\title{
Failure of feedback as a putative common mechanism of spreading depolarizations in migraine and stroke
}

Cite as: Chaos 18, 026110 (2008); https://doi.org/10.1063/1.2937120

Submitted: 21 February 2008 • Accepted: 06 May 2008 • Published Online: 27 June 2008

Markus A. Dahlem, Felix M. Schneider and Eckehard Schöll

\section{ARTICLES YOU MAY BE INTERESTED IN}

Controlling the onset of traveling pulses in excitable media by nonlocal spatial coupling and time-delayed feedback

Chaos: An Interdisciplinary Journal of Nonlinear Science 19, 015110 (2009); https:// doi.org/10.1063/1.3096411

\section{Mathematical approaches to modeling of cortical spreading depression}

Chaos: An Interdisciplinary Journal of Nonlinear Science 23, 046103 (2013); https:// doi.org/10.1063/1.4821955

Migraine generator network and spreading depression dynamics as neuromodulation targets in episodic migraine

Chaos: An Interdisciplinary Journal of Nonlinear Science 23, 046101 (2013); https:// doi.org/10.1063/1.4813815

\section{Scilight}

Summaries of the latest breakthroughs in the physical sciences 


\title{
Failure of feedback as a putative common mechanism of spreading depolarizations in migraine and stroke
}

\author{
Markus A. Dahlem, Felix M. Schneider, and Eckehard Schöll \\ Institut für Theoretische Physik, Technische Universität Berlin, \\ Hardenbergstraße 36, D-10623 Berlin, Germany
}

(Received 21 February 2008; accepted 6 May 2008; published online 27 June 2008)

\begin{abstract}
The stability of cortical function depends critically on proper regulation. Under conditions of migraine and stroke a breakdown of transmembrane chemical gradients can spread through cortical tissue. A concomitant component of this emergent spatio-temporal pattern is a depolarization of cells detected as slow voltage variations. The propagation velocity of $\sim 3 \mathrm{~mm} / \mathrm{min}$ indicates a contribution of diffusion. We propose a mechanism for spreading depolarizations (SD) that rests upon a nonlocal or noninstantaneous feedback in a reaction-diffusion system. Depending upon the characteristic space and time scales of the feedback, the propagation of cortical SD can be suppressed by shifting the bifurcation line, which separates the parameter regime of pulse propagation from the regime where a local disturbance dies out. The optimization of this feedback is elaborated for different control schemes and ranges of control parameters. (C) 2008 American Institute of Physics. [DOI: 10.1063/1.2937120]
\end{abstract}

During migraine and stroke, neurological symptoms occur representing pathological events that spread through the cerebral cortex. While these clinical observations have been known for a long time, only recently direct measurements were made. Two studies have revealed common spatio-temporal wave patterns, one using functional magnetic resonance imaging in a migraine patient ${ }^{1}$ and another using electrodes placed directly on the exposed cortical surface to record electrical activity in a stroke patient. ${ }^{2}$ The observed spatio-temporal patterns in the cortex constitute examples of excitable behavior that evidently emerges from pathological pathways. Spatial systems that exhibit the emergent property that activity breaks away from a local stimulation site are called excitable media. ${ }^{3,4}$ The capacity to propagate pulses is the distinguishing feature of excitability in spatial systems. As a mechanism for shifting the onset of excitability in a reaction-diffusion system we propose failure of nonlocal or noninstantaneous feedback control.

\section{INTRODUCTION}

The propagation of pathological states is a particular aspect within the complex bidirectional relation between migraine and stroke. ${ }^{5}$ Here we investigate this aspect, in particular, how cortical tissue when modeled as an excitable medium becomes susceptible to spreading events. Our knowledge about the mechanisms of propagation is still incomplete. It is generally believed that a common reactiondiffusion process, called cortical spreading depression (CSD) ${ }^{6-10}$ captures essential features of the observed spreading phenomena during migraine and stroke. What makes cortical tissue susceptible to CSD has not been determined. Since the smooth lissencephalic cortex of animals is much more susceptible to CSD than the convoluted cortex of hu- man, it was suggested that CSD in humans occurs very close to the onset of this emergent property. ${ }^{11,12}$

CSD is locally characterized by a nearly complete temporary breakdown of ion homeostasis, i.e., a stable stationary state (Fig. 1). ${ }^{9}$ Most electrophysiological changes follow a similar temporal course and return to normal after about $1 \mathrm{~min}$. A prominent signal is the slow negative potential shift $V_{e}$ during CSD. It led to the term spreading depolarization (SD) to classify this and related phenomena as depolarization waves in the cerebral cortex. ${ }^{13}$ Related phenomena are SD waves that contribute to progressive deterioration in regions surrounding a cortical infarct core in stroke patients. These $\mathrm{SD}$ waves are called peri-infarct depolarizations (PID). ${ }^{14} \mathrm{We}$ use the term SD for both cortical spreading depression and peri-infarct depolarizations in this paper. In this terminology spreading depolarization is the more general term. The respective form of SD depends mainly on differences in the energy state of cortical tissue. In particular, differences between CSD and PID concern increased blood flow compensating for the increased energy demands, which is typical for CSD occurring in healthy tissue during migraine, but is reduced or missing in PID during stroke.

Differences in brain regions, e.g., concerning the cytoarchitecture or the distribution of ion channel types, also modify SD. We disregard the detailed pathophysiological characterization of the process, because it is still incompletely understood. Instead, in this study, SD is modeled with a standard reaction-diffusion system of activator-inhibitor type. Differences are reflected in the choice of the parameter values of the system. We extend the reaction-diffusion model by different kinds of local feedback signals. We propose that one way to think of such local feedback signals is in terms of control. ${ }^{15}$ In this view, the feedback represents intrinsic cortical control mechanisms that reduce cortical susceptibility for CSD by stabilizing the physiological state of cortical tis- 


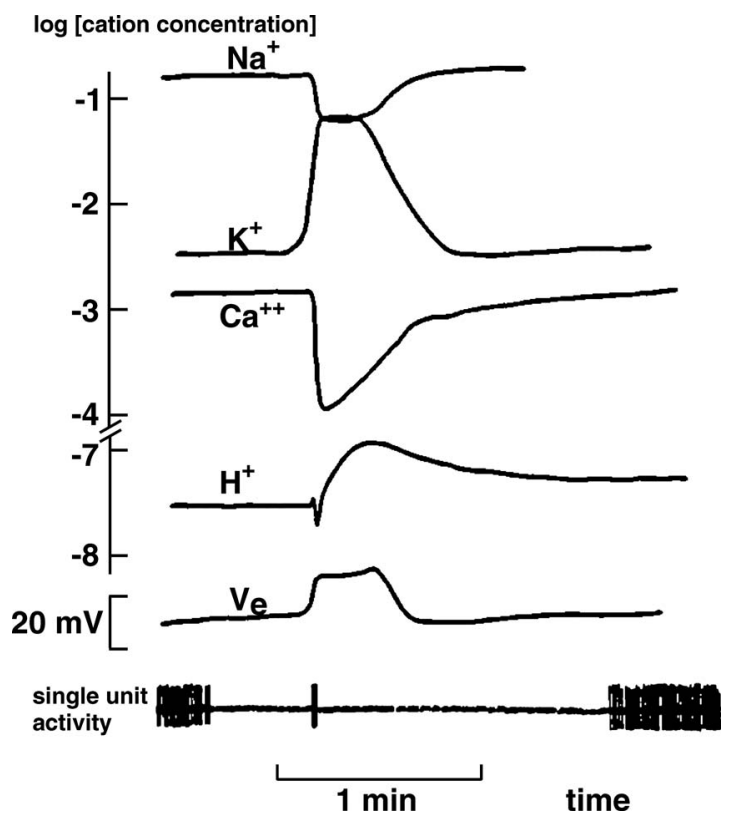

FIG. 1. Classical measurement of electrophysiological changes during cortical spreading depression. Upper four traces: logarithmic representation of changes in $\mathrm{Na}^{+}, \mathrm{K}^{+}, \mathrm{Ca}^{++}$, and $\mathrm{H}^{+}$concentration; lower two traces: extracellular potential shift $V_{e}$, and recording from a single neuron (single unit activity) vs time. [Figure by M. Lauritzen, "Pathophysiology of the migraine aura. The spreading depression theory," Brain 117, 199 (1994) (Ref. 9), reprinted by permission from Oxford University Press.]

sue. Consequently, their failure under certain pathological conditions leads to the emergence of CSD, e.g., attributed to an underlying cortical hyperexcitability in migraine ${ }^{16}$ or due to low energy levels in ischemic tissue during stroke. ${ }^{14}$

In this study, different feedback mechanisms with characteristic time and space scales are considered. Possible physiological basis of the feedback signals are motivated and discussed. We find that the excitability of the reactiondiffusion system can either increase or decrease. For each considered feedback scheme mainly the sign of the coupling strength determines whether feedback can suppress wave propagation, i.e., stabilize the homogeneous steady state of the tissue. A specific feedback scheme, in which activator and inhibitor variables are cross-coupled by nonlocal connections, indicates that opposed signs in the coupling strength for short-range and long-range connections are favorable for controlling the homogeneous steady state. This is a typical neuronal network connectivity pattern called Mexican-hat connectivity.

\section{THE MODEL}

While all evidence suggests that a reaction-diffusion process captures the essential features of SD, it is also clear that some features are oversimplified if we model SD with a standard reaction-diffusion mechanism of an activator-inhibitor type. To create a more accurate albeit still generic model, we extend the standard reaction-diffusion model by a feedback mechanism. We introduce the feedback signal pathway in two variations. First, we introduce nonlocal connections as a spatial coupling in addition to diffusion. This is motivated by the fact that high-frequency spikes in the population activity

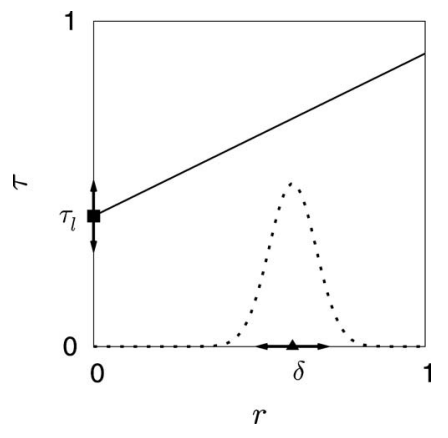

FIG. 2. Diagram of the spatial $(r)$ and temporal $(\tau)$ characteristics (solid line) of the feedback signal, which is used to extend the FitzHugh-Nagumo (FHN) reaction-diffusion model. One of the two FHN variables is fed back via connections with length $\delta$. The length is defined by the radial coordinate $r$ of a local polar coordinate system, which lies parallel to the cortical surface and has its origin located where the connection terminates. Due to a finite propagation velocity $c_{s}$ and a latency time $\tau_{l}$ of the feedback signal, a total time delay $\tau=\tau_{l}+c_{s}^{-1} \delta$ occurs. A kernel function $k(r)$ describes the spatial distribution of the connections, here exemplarily represented by a Gaussian (dashed). Two physiologically motivated limit cases of this general feedback type are considered: $\boldsymbol{\Delta}$ instant feedback along nonlocal connections, and local time-delayed feedback.

were observed up to millimeters ahead of the approaching front of SD. ${ }^{17,18}$ Second, we consider a localized time delayed feedback in the cortex, which can account for a slow feedback signal originating from the neurovascular coupling. Mathematically, these two extensions can be viewed as two limited cases of a general signal pathway (Fig. 2). Such a pathway serves as an additional coupling mechanism that is not included in a standard reaction-diffusion model. With this approach, we aim to describe universal features of excitable media modified by a feedback loop.

\section{A. Reaction-diffusion model}

We model the propagation of an initially localized breakdown of ion homeostasis in cortical tissue (Fig. 1) by an activator $u$ and an inhibitor $v$ as dynamic variables. They are coupled by kinetic reaction rates $f(u, v)$ and $g(u, v)$. We assume that only the activator species diffuses in the medium. The equations are

$$
\begin{aligned}
& \frac{\partial u}{\partial t}=f(u, v)+D \nabla^{2} u, \\
& \frac{\partial v}{\partial t}=\epsilon g(u, v) .
\end{aligned}
$$

The parameter $\epsilon$ is the time scale ratio in the local dynamics of activator and inhibitor variables. The local spatial coupling is introduced by the diffusion term in Eq. (1) with the diffusion coefficient $D$. Without loss of generality, the value of $D$ is normalized to unity. The choice of this value only scales the spatial coordinate.

In principle, there are two approaches to obtain the reaction rates $f(u, v)$ and $g(u, v)$. In a bottom-up approach, the reaction rates must be derived from a microscopic biophysical model of SD, where all major electrophysiological properties are represented. There is no consent so far on the mechanism of SD. Some models aim to provide a complete 
but rather local description of $\mathrm{SD},{ }^{19-21}$ that is, for processes occurring in a single neuron and its surrounding compartments. These models often introduce more than 20 dynamical variables. Therefore, a bottom-up approach with a subsequent reduction to two major activator and inhibitor agents seems to not be amenable. Nevertheless, it was shown that a two-species activator-inhibitor model can reproduce the spatio-temporal pattern of SD. ${ }^{11,12,22}$ Even a reactiondiffusion model with a single species was successfully introduced for the purpose of an order-of-magnitude estimate of the expected propagation velocity of SD. ${ }^{7,8}$ Such calculations follow essentially a top-down approach, which we also adopt here. The basic activator-inhibitor mechanism, which we describe in the next paragraph, can be expanded (top-down) to facilitate detailed investigation of further pathways and variables relevant to the study of specific questions concerning SD. Successful examples of such an approach are computational studies ${ }^{23,24}$ that supported a controversial hypothesis, namely, that cortical tissue surrounding an infarct core dies because of the metabolic stress imposed by multiple SD waves.

The variables $u$ and $v$ assume the roles of activator and inhibitor, respectively. Their kinetic functions $f(u, v)$ and $g(u, v)$ are given by a cubic nonlinearity and a linear function, respectively,

$$
\begin{aligned}
& f(u, v)=a\left(u-\frac{u^{3}}{3}\right)-v, \\
& g(u, v)=u-\beta-\gamma v
\end{aligned}
$$

with parameters $a, \beta$, and $\gamma$. This is the FitzHugh-Nagumo (FHN) system, which is widely used as a generic model of excitable media. ${ }^{25-27}$ In the result section, we will compare the simulated spatio-temporal patterns with the ones observed during SD. In particular, the pulse profile and velocity in the FHN system are related to the corresponding quantities in SD (Fig. 1). Following this approach, we can estimate realistic values of the parameters of the FHN system.

\section{B. FitzHugh-Nagumo system with feedback}

To extend the standard FHN reaction-diffusion model we assume that a nonlocal feedback signal $s(x, y, t)$ is coupled back into the medium at any point $(x, y)$ as

$$
\begin{aligned}
s(x, y, t)= & K \int_{0}^{2 \pi} \int_{0}^{\infty} k(r)\left[w\left(x+x_{r}, y+y_{r}, t-\tau\right)\right. \\
& -w(x, y, t)] d r d \phi,
\end{aligned}
$$

where $r$ and $\phi$ are the radius and the azimuthal angle, respectively, of a local polar coordinate system in $(x, y)$ that lies parallel to the cortical surface, i.e., $x_{r}=r \cos \phi$, and $y_{r}$ $=r \sin \phi$. The variable $w$ may be chosen as either the activator $u$ or the inhibitor $v$. The function $k(r)$ is the kernel function describing the spatial distribution of the pathway, which is typically peaked at a distance $r=\delta$, and $K$ is the coupling strength. The parameter $\tau=\tau_{p}(r)+\tau_{l}$, with $\tau_{p}(r)=r / c_{s}$, is a time delay composed of a latency $\tau_{l}$ and a propagation delay $\tau_{p}$. The latter is due to the finite signal propagation velocity $c_{s}$. A schematic diagram of the signal $s(x, y, t)$ is shown in Fig. 2. The feedback is, on the one hand, characterized by the parameter $\tau$, and the kernel function $k(r)$, i.e., $\delta$. We call this characterization the type of coupling of the feedback signal. On the other hand, there are two choices of $w$, namely the activator $u$ and the inhibitor $v$. Furthermore, each type can either be fed back to the activator $u$ or inhibitor $v$ rate equation, i.e., Eq. (1) or Eq. (2), respectively. This allows for four different combinations, named schemes, two self-coupling and two cross-coupling schemes for each type of coupling. The two self-coupling schemes are referred to as " $u u$ " if $w$ $=u$ and " $v v$ " if $w=v$, and the two cross-coupling schemes are referred to as " $v u$ " if $w=u$ and " $u v$ " if $w=v$. For example, in coupling scheme $u u$ the activator is used to compose the feedback signal $(w=u)$, which in turn is included in the activator rate equation (i.e., self-coupling). In a vector shorthand notation, the FHN system

$$
\frac{\partial \zeta}{\partial t}=F(\zeta), \quad \text { with } \zeta=\left(\begin{array}{l}
u \\
v
\end{array}\right)
$$

is replaced by

$$
\frac{\partial \zeta}{\partial t}=F(\zeta)+\sigma(x, y, t)
$$

where the coupling is given by

$$
\sigma=\left(\begin{array}{l}
s \\
0
\end{array}\right), \quad \text { or } \sigma=\left(\begin{array}{l}
0 \\
s
\end{array}\right)
$$

with $w=u$ or $w=v$, respectively.

Note that we have constructed the nonlocal feedback signal (5) such that it contains the difference between the remote and local values of $w$. As a consequence, the signal $s(x, y, t)$ tends to zero if the pattern is homogeneous and stationary. The motivation for such a feedback is that this choice eliminates the feedback signal in the case of successful suppression of wave propagation. The signal $s(x, y, t)$ can be viewed as an intrinsic noninvasive control because it preserves the homogeneous steady state as the fixed point of the uncontrolled $(K=0)$ FHN system. In this way, it can be compared with the common time-delayed feedback control method introduced by Pyragas. ${ }^{28}$ This method has been widely used with great success in problems in physics, chemistry, biology, and medicine ${ }^{15}$ including reactiondiffusion systems. ${ }^{29-33}$ In particular, it was demonstrated that it can be used to control the coherence and the time scales of noise-induced oscillations in a single FHN system ${ }^{34-36}$ and in two coupled excitable FHN systems ${ }^{37,38}$ as well as noiseinduced patterns in reaction-diffusion systems, ${ }^{39-43}$ and cause transition from oscillatory to excitable dynamics in a net of neural elements. ${ }^{44}$ This motivates our efforts to investigate whether a failure of such an intrinsic noninvasive control scheme can explain the onset of excitation spread in a spatially continuous FHN systems as a model of spreading pathological processes in the cortex during migraine and stroke. 


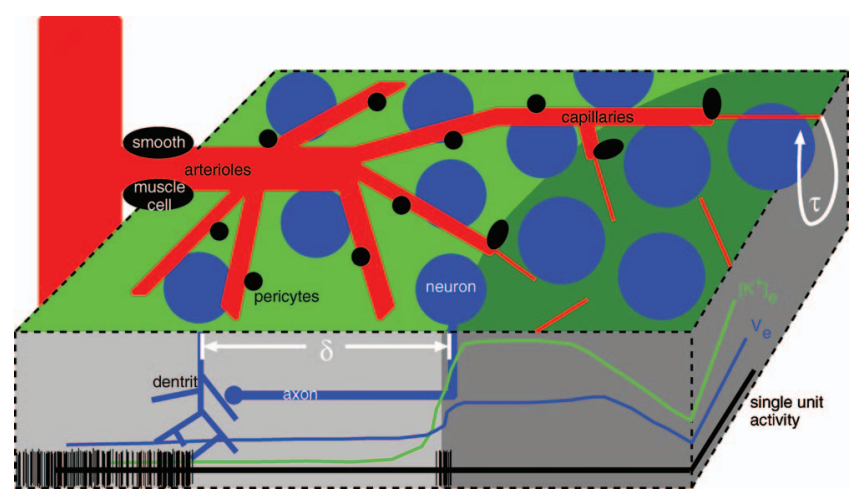

FIG. 3. (Color) Scheme of the cortical surface during cortical spreading depression. Red: blood vessels formed by endothelial cells. The cerebral blood flow starts at arterioles and leads to smaller capillaries, forming the capillary bed. Blue: neurons, green: glial cells. A pulse of cortical spreading depression moves from right to left, indicated by the shadowed region. The extracellular concentration changes of potassium $\left[\mathrm{K}^{+}\right]_{e}$, the extracellular potential shift $V_{e}$, and the single unit activity are schematically related to the pulse on the lateral front surface. Note that the size of the different cells types are enlarged by about a factor of 10 in relation to these measured signals. The two considered feedback mechanisms are also illustrated: (i) nonlocal instantaneous lateral connections of length $\delta$ between neurons (shown on the lateral front surface), (ii) a time delayed feedback control $(\tau)$ of the Pyragas-type due to local response of the neurovascular unit (indicated on the right lateral side surface).

\section{Instant feedback along nonlocal connections}

The first type of coupling that we consider is a limit case of Eq. (5). It is motivated by the observation of increased neuronal activity millimeters ahead of the approaching SD front. Although the cause and the effect of this activity remains unclear, it can be assumed that such activity is induced by SD via lateral cortical connectivity patterns (Fig. 3). In this case the signal propagation velocity $c_{s}$ is several orders of magnitude faster than the velocity $c$ of $\mathrm{SD}$, i.e., $c_{s} \gg c$ (50 $\mu \mathrm{m} \mathrm{s}^{-1}$ in physical units). Consequently, the propagation delay $\tau_{p}$ can be neglected. Furthermore, the time scale of typical changes in electrophysiological activity is much faster than the time scale of changes due to the breakdown of ion homeostasis. The latter time scale is of the order of seconds (Fig. 1), which is also in agreement with our simulations of the FHN system if we dimensionalize the model equations (see Sec. III below), while the former time scale, i.e., the delay due to chemical or electrical transmission at synapses, is of the order of milliseconds. Therefore, it is reasonable to assume that the latency $\tau_{l}$ of $s(x, y, t)$ is much smaller than the time scale of SD and that $\tau_{l}$ can also be neglected. This assumption can fail for metabotropic ion channels, like metabotropic glutamate receptors, which have increased open probabilities in the range of seconds after their activation. However, for ionotropically mediated activity we assume that $\tau_{p} \approx 0$ and $\tau_{l} \approx 0$, and therefore that the additional coupling signal $s(x, y, t)$ is, in this case, an instantaneous process $(\tau=0)$. Furthermore, we assume that the connectivity pattern is rather localized around the typical coupling length $\delta$, and therefore, restricting ourselves to one spatial dimension, $x$, we approximate the kernel function by $\delta$-functions $k(r) \approx \delta(x-\delta)+\delta(x+\delta)$. Taking these limits, Eq. (5) reduces to

$$
s_{\delta}(x, t)=K[w(x-\delta, t)-2 w(x, t)+w(x+\delta, t)] .
$$

Note that Eq. (9) only in the limit $\delta \rightarrow 0$ becomes a standard diffusion term. However, for large $\delta$, as considered here, it adds a novel type of nonlocal spatial coupling to the FHN model.

\section{Local time-delayed feedback}

The second type is also a limit case of Eq. (5). We consider a scenario that takes into account the coupling within the neurovascular unit. Therefore, it is particularly important for the dynamics of peri-infarct depolarizations during stroke progression. The cerebral blood flow $(\mathrm{CBF})$ is tightly regulated by the neurovascular unit to meet the brain's metabolic demands. These demands are extremely high during SD. The dynamics of the neurovascular unit are governed by various types of cells (Fig. 3). The interaction takes place between endothelial cells building the vessel walls, mural cells controlling vessel diameter, and glia and neurons. For the purpose of our simplified scheme, that is, describing universal features of reaction-diffusion models of SD modified by an additional signal pathway, we concentrate on some key features of blood flow regulation that occur within the time and space scale given by a single passage of SD and that mimic the influence of the neurovascular unit.

The local CBF starts at arterioles, that is, blood vessels that extend and branch out from an artery and lead to capillaries. Capillaries are the smallest vessels, measuring $5-10 \mu \mathrm{m}$ in diameter. They form the capillary bed, a local network supplying the brain tissue. The CBF is regulated by two types of mural cells. Smooth muscle cells regulate arterioles, and pericytes regulate capillaries. The majority of the innervation of cerebral blood vessels terminate near capillaries suggesting that blood flow control is initiated in capillaries. ${ }^{45}$ Therefore, we need to consider that increased activity during SD produces an initially localized hemodynamic response evoked by pericytes. To describe the action of the neurovascular unit on SD, the temporal and spatial distribution of the hemodynamic response needs to be considered.

First, we consider the spatial distribution of the hemodynamic response, which is determined by the vascular architecture. A precise spatial coordination of segmental vascular resistance is needed to effectively increase blood flow in a larger cortical area. Propagated vascular response signals are utilized to achieved this. It was shown that after the pericytes evoke a local capillary constriction, a pulse of constriction propagates at about $2 \mu \mathrm{m} \mathrm{s}^{-1}$ to distant pericytes. ${ }^{45}$ Furthermore, a dilatory signal mediated by release of vasoactive agents propagates from metabolically active cells and evokes a remote response in upstream precapillary arterioles. ${ }^{46}$ Considering the slow velocity of $2 \mu \mathrm{m} \mathrm{s}^{-1}$ we can assume that global effects mediated by arterioles take place behind SD, i.e., after its passage, and therefore do not have a direct influence on the propagation of the wave front having passed. We assume that the response of the capillaries is rather localized, so that we can approximate the kernel function $k(x) \approx \delta(x)$. Consequently there is only a local response. Still 
there exists a latency delay $\tau_{l}$ due to slow metabolic effects when a local hemodynamic response is evoked by pericytes. Taking these limits, Eq. (5) now reduces to

$$
s_{\tau}(x, t)=K[w(x, t-\tau)-w(x, t)],
$$

which is identical with the noninvasive time-delayed feedback signal first introduced by Pyragas ${ }^{28}$ for chaos control. This control method will now be applied to travelling pulses in excitable media. The latency delay time $\tau$ will be varied in our simulations.

Note that during stroke progression, the recruitment of peri-infarct tissue into the infarct core is probably mediated by wave trains of peri-infarct depolarizations. ${ }^{13}$ Such periodic wave patterns are likely to be influenced by large scale hemodynamic responses. After the leading SD has initiated a slow propagating wave of constriction, ${ }^{45}$ subsequent waves in the wave train formation can be influenced by this. This is beyond the scope of our study. To model a single SD pulse, we include the neurovascular unit by a localized timedelayed feedback signal $s_{\tau}(x, t)$ evoked from the change in blood flow which in turn is caused by pericytes in response to changes in neural activity.

\section{RESULTS}

It was suggested that $\mathrm{SD}$ in humans occurs close to the bifurcation of the onset of spreading activity in excitable media. ${ }^{11,12}$ Therefore, we first determine the location of this onset in the FHN system. Then we estimate the parameters of the FHN system from experimental data on SD (Fig. 1) and compare them with corresponding quantities in brain tissue. Finally, we investigate how the onset of excitability is influenced by the two types of coupling, $s_{\delta}(x, t)$ and $s_{\tau}(x, t)$, introduced in the previous section, in particular, how the onset is influenced by the newly introduced spatial and temporal scales $\delta$ and $\tau$, respectively. To achieve this, we first consider the uncontrolled FHN system in the regime where sustained pulse propagation is possible and investigate whether or not the additional coupling schemes suppress pulse propagation. In this context, we can view the coupling scheme as a control scheme with the control goal to stabilize the homogeneous steady state. ${ }^{47-49}$ Second, we choose parameter pairs $\left(K_{0}, \delta_{0}\right)$ and $\left(K_{0}, \tau_{0}\right)$ that suppress pulse propagation and determine the location of the onset of propagation in the FHN system with these coupling schemes. Furthermore, we determine the location of the onset of propagation in the FHN system for those cases where the coupling schemes cannot suppress pulse propagation.

The spread of an initially local activity arises in the system described by Eqs. (1)-(4) if a critical parameter value is crossed above which the medium is susceptible for sustained propagating excitation patterns. ${ }^{3,50-52}$ In a $1 \mathrm{D}$ medium this border is obtained by finding the regime where stationary solutions exist in a co-moving frame for specific activator and inhibitor profiles. In the co-moving frame $\xi=x+c t$, the coupled partial differential equations for activator and inhibitor variables Eqs. (1) and (2) transform to a system of second order ordinary differential equations for $U(\xi)$ and $V(\xi)$,

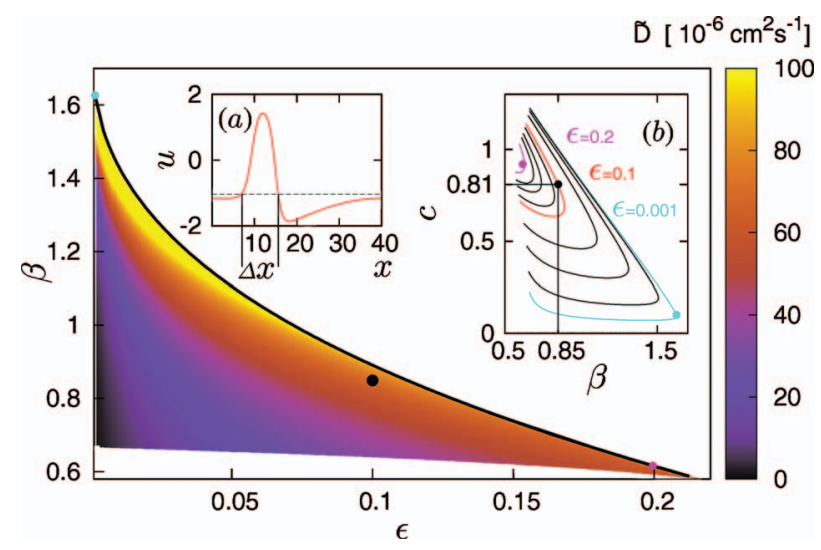

FIG. 4. (Color) Parameter space of the FHN system at $\gamma=0.5$ and $a=1$. The dimensionalized diffusion coefficient is color-coded as a function of the time scale ratio $\epsilon$ and the excitability threshold parameter $\beta$. The excitable regime (color-shaded) and the nonexcitable regime (white) are separated by the boundary of propagation (thick black line). Above this boundary traveling pulse solutions do not exist. Inset (a) shows a typical pulse profile corresponding to the black dot in the main figure. Inset (b) shows homoclinic orbits of the FHN system in the co-moving frame with velocity $c$ as a function of $\beta$ for different values of $\epsilon$. In this diagram, the location of the boundary of propagation is determined by the value of $\beta$ at the turning point of each curve and the corresponding $\epsilon$ value, exemplarily shown by cyan and magenta dots for $\epsilon=0.001$ and $\epsilon=0.2$, respectively, in inset (b) and the main figure.

$$
u(x, t)=U(\xi), \quad v(x, t)=V(\xi),
$$

which can be further transformed to a system of coupled first order ordinary differential equations of three variables, $U(\xi)$, $V(\xi), W(\xi) \equiv \partial U(\xi) / \partial \xi{ }^{53}$ Due to this transformation, $c$, the propagation velocity, is introduced as an additional parameter. In this system, homoclinic orbits can be found using a continuation method. ${ }^{54}$ They correspond to travelling pulse solutions in Eqs. (1) and (2) (Fig. 4, inset b). The transition into a region where homoclinic orbits exist marks a bifurcation of co-dimension one. In the parameter space of excitable media this bifurcation separates the regime supporting travelling pulses from the nonexcitable regime. This border is shown in Fig. 4 in the parameter plane $(\epsilon, \beta)$ at the section $a=1$ and $\gamma=0.5$. As expected, the transition into the nonexcitable regime is achieved by increasing either $\beta$ or $\epsilon$. Increasing $\beta$ increases the threshold of the excitable medium, while increasing $\epsilon$ changes the time scale ratio between the dynamics of activator and inhibitor, with larger values of $\epsilon$ corresponding to faster inhibitor dynamics.

\section{A. Estimate of space and time scales}

The main reason to introduce spatial and temporal units in our FHN system is to obtain a better understanding of the typical values of the spatial and temporal coupling scales, $\delta$ and $\tau$, respectively. Furthermore, the value of the diffusion coefficient $D$ can be determined if we introduce dimensional units in the FHN system. Note that $D$ is not a bifurcation parameter. In the nondimensionalized FHN system Eqs. (1)-(4), $D$ can assume any value without changing the dynamics in a qualitative way. For example, changing $D$ has no effect on the location of the propagation boundary shown in Fig. 4. Varying $D$ is equivalent to scaling space units by 
$D^{-1 / 2}$. Therefore, the physical value of the diffusion coefficient $D$ can be determined if we match the patterns obtained in the FHN system with the ones observed experimentally during SD.

First, we define characteristic space and time units, $x_{0}$ and $t_{0}$, respectively. This is done with respect to the spatiotemporal patterns of the uncontrolled $(K=0)$ FHN system. These characteristic units are chosen such that the patterns calculated from the nondimensionalized Eqs. (1)-(4) match the measured ones of Fig. 1. Introducing dimensional space $(X)$ and time $(T)$ variables by $x=X / x_{0}$ and $t=T / t_{0}$, the FHN system Eqs. (1) and (2) assumes its dimensional form with the dimensionalized diffusion coefficient $\widetilde{D} \equiv x_{0}^{2} / t_{0}$.

Now we compare the simulated pulse width $\Delta x$ and duration $\Delta t$ with the measured pulse width $\Delta X$ and measured duration $\Delta T$ (Fig. 1, $\Delta T \approx 20 \mathrm{~s}$ ). Using the typical measured velocity of $\mathrm{SD} \quad C \approx 50 \mu \mathrm{m} \mathrm{s}^{-1}$, we obtain $\Delta X=C \Delta T$ $\approx 0.1 \mathrm{~cm}$. The simulated pulse width $\Delta x$ and duration $\Delta t$ are related by $\Delta x=c \Delta t$ with the nondimensionalized propagation velocity $c$, which appears as a parameter in Eq. (11) [e.g., for $\epsilon=0.1$ and $\beta=0.85: \Delta x \approx 8.7$ in Fig. 4 (inset a) and $c$ $\approx 0.81$ in Fig. 4 (inset b), hence $\Delta t \approx 10.70]$. Hence we infer the space and time units $x_{0}=\Delta X / \Delta x \approx 115 \mu \mathrm{m}$ and $t_{0}$ $=\Delta T / \Delta t \approx 1.9 \mathrm{~s}$, respectively, which yields a diffusion coefficient $\widetilde{D}=x_{0}^{2} / t_{0} \approx 70 \times 10^{-6} \mathrm{~cm}^{2} \mathrm{~s}^{-1}$, which is a reasonable value. Note that the pulse width $\Delta x$, the velocity $c$, and hence $x_{0}, t_{0}$, and $\tilde{D}$ depend upon the parameters $\beta$ and $\epsilon$.

In Fig. 4 the obtained values for the diffusion coefficient $\widetilde{D}$ for each pair $(\epsilon, \beta)$ are shown in color coding. It ranges from $5 \times 10^{-6}$ to $100 \times 10^{-6} \mathrm{~cm}^{2} \mathrm{~s}^{-1}$ in the main part of the parameter space. As expected, low values are obtained in regions far away from the boundary of propagation. There the excitability of the system is high. On the other side, close to the propagation boundary, the values of $\widetilde{D}$ are higher. In this regime, the medium is weakly excitable or subexcitable. The correlation between the value of the obtained diffusion coefficients $\widetilde{D}$ and the excitability regimes requires some further clarification, which will be addressed in the discussion. However, note that the values of $\widetilde{D}$ in the parameter plane $(\epsilon, \beta)$ at the section $a=1$ and $\gamma=0.5$ are in the range of expected values for a diffusing substance participating in the mechanism of SD, as discussed in the literature. ${ }^{7,8,12,55,56}$

\section{B. Control of spreading depressions by $s_{\delta}(x, t)$ and $s_{\tau}(x, t)$}

How does the additional coupling $s(x, t)$ change the FHN system? To answer this, we perform simulations with a variety of FHN systems in the parameter plane $(\epsilon, \beta)$ at $a=1$ and $\gamma=0.5$. The systems are chosen close to the propagation boundary (Fig. 4). We include both types of coupling, the instant feedback along nonlocal connections $s_{\delta}(x, t)$, described in Sec. II B 1, and the local time-delayed feedback $s_{\tau}(x, t)$, described in Sec. II B 2. Both types are considered separately. For each type of coupling there are four coupling schemes, two self-coupling schemes $(u u, v v)$ and two crosscoupling schemes $(u v, v u)$.
To obtain the influence of the coupling schemes on the FHN system, we start each individual simulation with a stable pulse profile of the reaction-diffusion system obtained without the coupling signal $(K=0)$. Then, for $K \neq 0$, we determined whether or not the pulse propagation is terminated. If the propagation is suppressed, we also determine how long the pulse can still propagate before it disappears. This distance defines the volume of tissue at risk (TAR), referring to the risk of cortical tissue surrounding a local pathological core of being recruited into the disturbed state. ${ }^{57}$ This TAR value is taken as a measure of the efficiency of the coupling scheme as a control method.

\section{Boundary of pulse propagation}

First, we consider the four nonlocal schemes in $s_{\delta}(x, t)$, i.e., instant coupling along nonlocal connections (Sec. II B 1). Regions in which pulse propagation is suppressed are shown in Figs. 5(a)-5(d). The TAR value is given by a color code in this regions. For the two cases where we introduce self-coupling [Fig. 5(a) $(w=u)$ and Fig. 5(d) $(w=v)$ ], mainly the sign of the coupling constant $K$ determines the success of the coupling scheme. Pulse propagation cannot be suppressed with negative values of $K$. This can be intuitively understood, if we consider only the effect of the signal $s_{\delta}(x, t)$ on the homogeneous steady state. A small disturbance from the homogeneous state is destabilized by $s_{\delta}(x, t)$ if $K$ $<0$ and stabilized if $K>0$. Optimal values of $\delta$ are obtained for $w=u$ at $\delta \approx \Delta x$ and for $w=v$ at $\delta \approx 0.5 \Delta x$. The picture changes for the two schemes with cross-coupling [Fig. 5(b) $(w=v)$ and Fig. 5(c) $(w=u)]$. Successful suppression of pulse propagation in these cases depends on both the coupling strength $K$ and the coupling length $\delta$. The change in the sign of $K$ occurs at $\delta \approx \Delta x$. If $\delta<\Delta x, K$ must be positive (negative) if the coupling term $s_{\delta}(x, t)$ is fed into the inhibitor (activator) balance Eq. (2) or (1), respectively, and vice versa for $\delta>\Delta x$. In other words, the short and long range control domains have opposite signs of $K$. Optimal values of $\delta$ in the short range control domain are obtained at $\delta \approx 0.5 \Delta x$.

Next, we consider the local time-delayed feedback coupling (Sec. II B 2). In each of the four coupling schemes control is only achieved if $K$ is either positive or negative [Figs. 5(e)-5(h)]. For positive $K$, the pulse propagation is suppressed if the signal is coupled into the activator $u$. The two domains in the $(K, \tau)$ plane, where pulse propagation is suppressed, are of comparable size [Fig. 5(e) (self-coupling) and Fig. 5(f) (cross-coupling)]. For negative $K$, the pulse propagation is suppressed if the signal is coupled into the inhibitor $v$. In this case, the cross-coupling scheme [Fig. $5(\mathrm{~g})]$ has a domain of comparable size and shape as for cases shown in Figs. 5(e) and 5(f). The domain for the selfcoupling scheme is much smaller with only large values of TAR, i.e., less efficient suppression of pulse propagation [Fig. 5(h)]. For all four cases, the optimal delay time is $\tau$ $\approx 0.5 \Delta t$. 

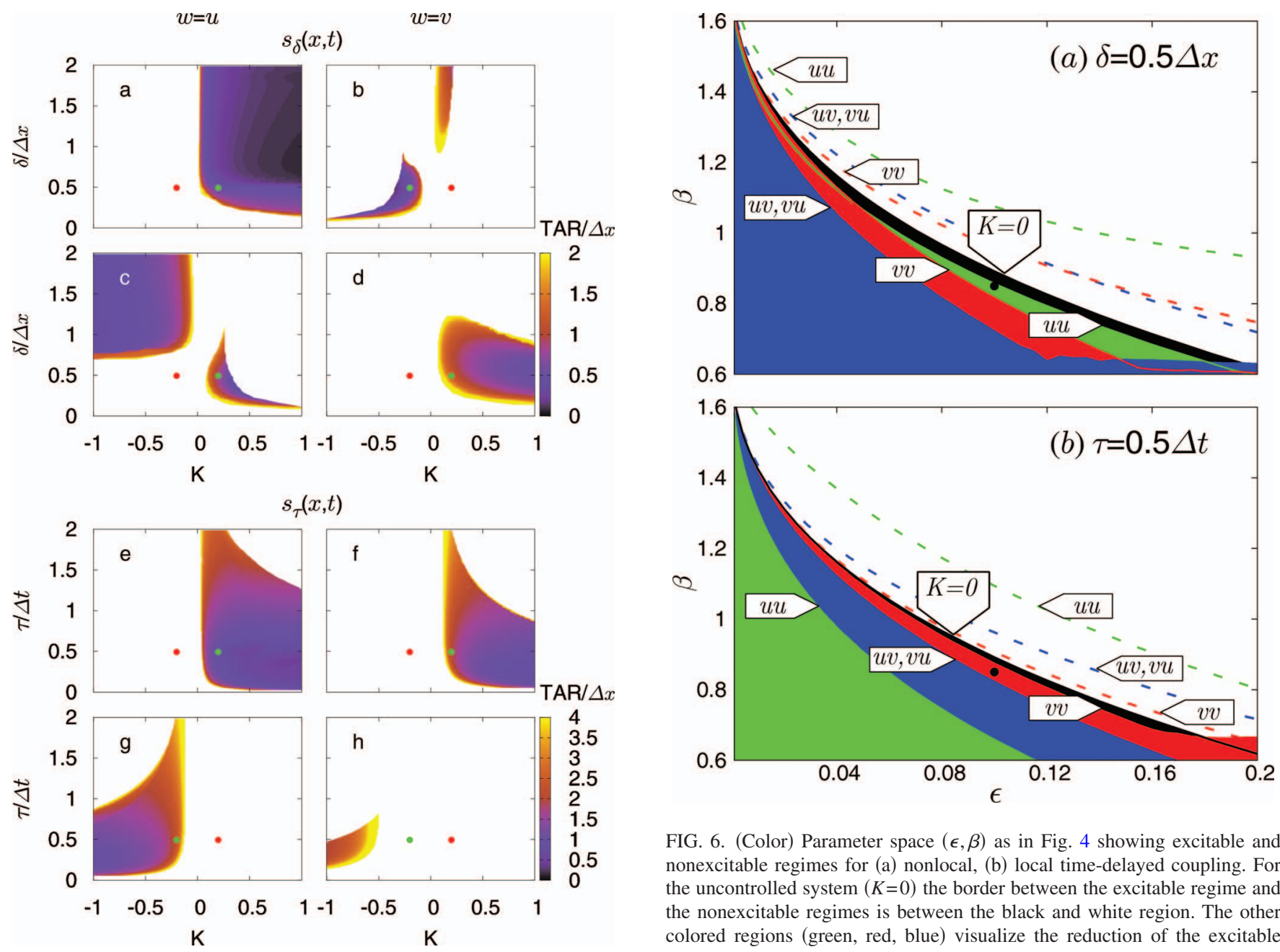

FIG. 6. (Color) Parameter space $(\epsilon, \beta)$ as in Fig. 4 showing excitable and nonexcitable regimes for (a) nonlocal, (b) local time-delayed coupling. For the uncontrolled system $(K=0)$ the border between the excitable regime and the nonexcitable regimes is between the black and white region. The other colored regions (green, red, blue) visualize the reduction of the excitable regime by the different coupling schemes as indicated by the location of the green dots in Fig. 5, i.e., $K$ is chosen as $K=0.2$ [uu, uv, vv in (a); uu, vu in

FIG. 5. (Color) Control planes for the four coupling schemes of the two types of feedback $s_{\delta}(x, t)$ (a)-(d) and $s_{\tau}(x, t)(\mathrm{e})$-(h). (a), (e) Self-coupling of the activator signal $(u u)$; (b), (f) self-coupling of the inhibitor signal (vv); (c), (g) cross-coupling with the feedback signal composed of the activator $(w=u)$ and fed back to the inhibitor rate equation (uv); (d), (h) crosscoupling with the inverse configuration as compared to (c), (g), i.e., $(v u)$. The parameter values of the FHN system are $\epsilon=0.1, \beta=0.85, a=1$, and $\gamma$ $=0.5$. This system is close to the propagation boundary (see black dot in Fig. 4). Regions in which pulse propagation is suppressed by the feedback signal are shown color coded by the tissue at risk (TAR) value, that is, the volume of tissue recruited into the pathological state before the pulse dies out. Low TAR values indicate that the feedback signal is more efficient. Optimal values, i.e., low TAR values, for $\delta$ and $\tau$ are mainly located at about 0.5 times the activator pulse width and duration, respectively (see text). Red and green dots mark control parameter values $(K, \delta)$ and $(K, \tau)$ for which the shift in the pulse propagation boundary is calculated in Fig. 6.

\section{Shift in the onset of excitability by $s_{\delta}(x, t)$ and $s_{\tau}(x, t)$}

We now investigate the shift in the location of the propagation boundary (Fig. 4) caused by the four coupling schemes of both types of coupling $s_{\delta}(x, t)$ and $s_{\tau}(x, t)$. This boundary separates the excitable regime from the nonexcitable regime. Intuitively it is clear that the suppression of pulses shifts the propagation boundary towards the excitable regime of the system with $K=0$. If we consider the coupling schemes as control mechanisms, such a shift manifests itself as a successful control strategy that reduces the excitability of the system. opposite sign of $K$ (red dots in Fig. 5) the excitable regime increases indicated by the dashed lines.

First, we consider the nonlocal type of coupling $s_{\delta}(x, t)$. The parameter values for $s_{\delta}(x, t)$ are set to $K=0.2$ and $K$ $=-0.2$, and $\delta=0.5 \Delta x$. For each of the four coupling schemes with $\delta=0.5 \Delta x$ only one value of $K$, either $K=0.2$ or $K$ $=-0.2$, suppresses pulse propagation (marked by green dots in Fig. 5). With this choice and depending upon the four coupling schemes, the control signal $s_{\delta}(x, t)$ lies either close to optimal $\delta$ values or on the opposite side, mirrored along the $K=0$ axis (marked by red dots in Fig. 5), a location where the control goal is not achieved. In the cases of the two cross-coupling schemes, the value $K=-0.2$ for $w=v$ [Fig. 5(b)] and the value $K=0.2$ for $w=u$ [Fig. 5(c)] lies in the center of the control domain with the short range connections. If we consider the four cases in which pulses are suppressed, the location of the propagation boundary is shifted into the excitable regime, indicated by differently colored regions in Fig. 6(a). It is evident that the $u v$ and $v u$ cross coupling schemes are most efficient. Note that for the least efficient coupling scheme $(u u)$ the propagation boundary [edge of the green region in Fig. 6(a)] is actually not shifted beyond the point $\epsilon=0.1$ and $\beta=0.85$ (black dot). This seems to be contradictory with the control domain shown in Fig. 
5(a), but is consistent with the definition of the propagation boundary as a bifurcation line. Beyond this bifurcation line, traveling pulse solutions do not exist, whereas in Fig. 5(a), only states with a specific traveling pulse solution with a specific amplitude (the one of the uncontrolled system) is suppressed. This example illustrates the different nature of the information shown in Figs. 5 and 6. In the four cases with the opposite sign of the parameter value $K$, i.e., where pulses are not suppressed, the propagation boundary is shifted towards the nonexcitable regime of the uncontrolled $(K=0)$ FHN system [dashed lines in Fig. 6(a)].

Second, we consider the local, time-delayed type of coupling $s_{\tau}(x, t)$. The parameter values for $s_{\tau}(x, t)$ are set to $K$ $=0.2$ and $K=-0.2$, and $\tau=0.5 \Delta t$. To provide a comparison with the former type of coupling $s_{\delta}(x, t)$, we also choose the short range domain [as for $s_{\delta}(x, t)$ in Figs. 5(b) and 5(c)] as the reference point and use these values of $K$ also for $s_{\tau}(x, t)$. As before they are marked by green dots in Figs. 5(e) $-5(\mathrm{~g})$ if control is successful and red otherwise. Only for the scheme $(v v)$ [Fig. 5(h)] both types of coupling for $K=-0.2$ and $K$ $=0.2$ lie outside the control domain. Since the type with $K$ $=-0.2$ is closer to a control domain, this is marked green, and correspondingly its propagation boundary is slightly pulled back as indicated by the red region in Fig. 6(b). Again we find a similar pattern as for the nonlocal type of coupling $s_{\delta}(x, t)$. The coupling schemes that suppress pulse propagation reduce excitability [colored regions in Fig. 6(b)] and the other ones increase excitability (dashed lines). Note that also for the coupling scheme $(v v)$ this pattern occurs, with the only difference that the propagation boundary [edge of the red region in Fig. 6(b)] is not shifted beyond the point $\epsilon$ $=0.1$ and $\beta=0.85$ (black dot), which is in agreement with the control domain shown in Fig. $5(\mathrm{~g})$.

\section{DISCUSSION AND CONCLUSIONS}

We have shown that a weakly excitable reactiondiffusion system can be shifted across the boundary of pulse propagation (Fig. 6) by various types of feedback and coupling schemes. In particular, we use nonlocal feedback $s_{\delta}(x, t)$ and time-delayed feedback $s_{\tau}(x, t)$. On the one hand, this is motivated by physiological considerations (Fig. 3). On the other hand, these two distinguished types allow for a clear separation of the effect of the introduced space scale $\delta$ and time scale $\tau$ upon the spatio-temporal pattern of SD. We have shown that these scales $\delta$ and $\tau$ are closely related. The reason is, of course, that the feedback is applied to control of travelling pulses. The velocity of SD relates space to time scales. Their numerical values can be compared after normalizing them with the pulse width $\Delta x$ and pulse duration $\Delta t$, respectively. The common effect of both types of feedback on the traveling pulse is then reflected in the similar location of optimal control around half the pulse width and pulse duration for $s_{\delta}(x, t)$ and $s_{\tau}(x, t)$, respectively (Fig. 5). Therefore, the feedback mechanism, as given in a general form in Eq. (5), and illustrated in Fig. 2, provides a generic mechanism to modulate excitability in reaction-diffusion systems even if we consider only its two limit cases.
We have used the FHN model as a reaction-diffusion system to demonstrate the modulation of excitability by feedback. The FHN mechanism represents excitable media exhibiting excitability of type II. Furthermore, we have constrained our investigations to the section $a=1$ and $\gamma=0.5$ in the four-dimensional parameter space of this model. Both restrictions, excitability of type II and this choice within the parameter space, will now be discussed. Type II excitability is related to a Hopf bifurcation, which is characterized by the fact that a periodic state bifurcates with a nonzero frequency ${ }^{58}$ Within an exponentially small range of either $\beta$ or $\epsilon$ after the Hopf bifurcation a transition occurs, called canard explosion, from a state with small amplitude oscillations to a large amplitude relaxation oscillation. The canard explosion produces the threshold behavior (all-or-none) of the system needed to exhibit excitable behavior. Another typical bifurcation scenario leading to excitability is that the periodic state appears at a zero frequency, ${ }^{58,59}$ called type I excitability. This can be modeled by a saddle-node bifurcation on a limit cycle or saddle-node infinite period bifurcation (SNIPER). ${ }^{41,60}$

It is not clear which type of excitability is the basis of SD. However, experiments with retinal spreading depression indicate excitability of type II behavior. ${ }^{61}$ The extracellular potassium concentration $\left(\left[\mathrm{K}^{+}\right]_{e}\right)$ was increased stepwise. Above a usually well preserved ceiling level of $\left[\mathrm{K}^{+}\right]_{e}$ $=10 \mathrm{mM},{ }^{62}$ a rather spontaneous onset of oscillations with finite period was observed. Due to the presence of noise and a rather large step width of $\Delta\left[\mathrm{K}^{+}\right]_{e}=2 \mathrm{mM}$, a definite conclusion is not yet possible. However, we assume that our results hold in principle also for excitable media in which each local element is of excitability type I. The difference is manifest only close to the bifurcating state of the individual excitable element. In an excitable media, that is in a spatially extended system, excitability is defined by the distinguishing feature that the excited state breaks away from a local stimulated area due to transport, which usually involves diffusion. The bifurcating state of the individual elements seems therefore less important than the saddle-node bifurcation that leads to the emergence of traveling pulses (Fig. 4, inset b).

The FHN system is the most generic type that shows excitability of type II, because the activator equation, Eq. (1), has a cubic nonlinearity, which is generic for bistability. ${ }^{63,64}$ Since we aim to describe generic features, let us briefly comment on the choice of the two parameters that we have fixed $(a=1$ and $\gamma=0.5$ ). With this choice, the value of the diffusion coefficient in the main part of the remaining section of the parameter space is between $5 \times 10^{-6} \mathrm{~cm}^{2} \mathrm{~s}^{-1}$ and 100 $\times 10^{-6} \mathrm{~cm}^{2} \mathrm{~s}^{-1}$ (Fig. 4). The diffusion coefficient of $\left[\mathrm{K}^{+}\right]_{e}$, a substance often related to the activator or at least to the species by which SD propagates, is about $20 \times 10^{-6} \mathrm{~cm}^{2} \mathrm{~s}^{-1}$ in aqueous solution. ${ }^{65}$ There are two counteracting effects that can significantly change this value in cortical tissue. First, due to the porous geometrical structure and small volume fraction of the extracellular space, which is similar to a soap phase, the apparent diffusion coefficient of $\left[\mathrm{K}^{+}\right]_{e}$ in brain tissue is estimated to $7.2 \times 10^{-6} \mathrm{~cm}^{2} \mathrm{~s}^{-1}$. ${ }^{55}$ Second, the possibility that $\left[\mathrm{K}^{+}\right]_{e}$ enters glial cells in one place and leaves elsewhere provides a form of facilitated diffusion that is es- 
timated to be up to five times more important than diffusion in the extracellular space. ${ }^{8}$ In summary, a generic model that simulates traveling pulses with a diffusion coefficient $\tilde{D}$ of reasonable order of magnitude seems to be appropriate.

We propose that a failure of feedback provides a common mechanism of the emergence of spreading depolarizations in migraine and stroke. In our generic approach, our concern is not to identify the species behind activator, inhibitor, and feedback signals, but rather to describe spatial and temporal properties of their interaction that leads to the emergence of travelling pulses. This approach focuses on the clinically important question whether a local breakdown of homeostasis recruits further tissue into the excited (pathological) state or remains restricted to the initial focus. The recruitment of tissue can only be studied in spatially extended systems, in which a pulse propagation boundary can be defined (Fig. 4). The existence of the propagation boundary has consequences for therapeutic approaches that aim at an effective reduction of excitability to hold the tissue below the bifurcating state and with that limit the tissue at risk. ${ }^{57}$ By contrast, biophysical models of depolarizations in a single neuron and its surrounding extracellular and glial compartments infer statements about local individual elements of an excitable medium, which complement our generic approach. For instance, a study of the local SD dynamics has concluded that the key to normal stability of cortical tissue is the effective regulation of $[\mathrm{K}+]_{e}$ by the neuron's $\mathrm{Na}-\mathrm{K}$ ion pump and the glia-endothelial system. ${ }^{20}$ Future investigations have to combine these two approaches toward a better understanding of spreading depolarizations.

\section{ACKNOWLEDGMENTS}

This work was supported by DFG in the framework of Sfb 555. The authors would like to thank H. Engel, H. R. Wilson, and K. Showalter for fruitful discussions.

${ }^{1}$ N. Hadjikhani, M. Sanchez Del. Rio, O. Wu, D. Schwartz, D. Bakker, B. Fischl, K. K. Kwong, F. M. Cutrer, B. R. Rosen, R. B. Tootell, A. G. Sorensen, and M. A. Moskowitz, "Mechanisms of migraine aura revealed by functional MRI in human visual cortex," Proc. Natl. Acad. Sci. U.S.A. 98, 4687 (2001).

${ }^{2}$ M. Fabricius, S. Fuhr, R. Bhatia, M. Boutelle, P. Hashemi, A. J. Strong, and M. Lauritzen, "Cortical spreading depression and peri-infarct depolarization in acutely injured human cerebral cortex," Brain 129, 778 (2006). ${ }^{3}$ A. T. Winfree, "Varieties of spiral wave behavior: An experimentalist's approach to the theory of excitable media," Chaos 1, 303 (1991).

${ }^{4}$ A. S. Mikhailov and K. Showalter, "Control of waves, patterns and turbulence in chemical systems," Phys. Rep. 425, 79 (2006).

${ }^{5}$ M. G. Bousser and K. M. Welch, "Relation between migraine and stroke," Lancet Neurol. 4, 533 (2005).

${ }^{6}$ A. A. P. Leão, "Spreading depression of activity in the cerebral cortex," J. Neurophysiol. 7, 359 (1944).

B. Grafstein, "Neural release of potassium during spreading depression," in Brain Function, Cortical Excitability, and Steady Potentials, edited by M. A. B. Brazier (University of California Press, Berkely, 1963), pp. 87-124.

${ }^{8}$ A. R. Gardner-Medwin, "Possible roles of vertebrate neuroglia in potassium dynamics, spreading depression and migraine," J. Exp. Biol. 95, 111 (1981).

${ }^{9}$ M. Lauritzen, "Pathophysiology of the migraine aura. The spreading depression theory," Brain 117, 199 (1994).

${ }^{10}$ H. Martins-Ferreira, M. Nedergaard, and C. Nicholson, "Perspectives on spreading depression," Brain Res. Rev. 32, 215 (2000).
${ }^{11}$ M. A. Dahlem and E. P. Chronicle, "A computational perspective on migraine aura," Prog. Neurobiol. 74, 351 (2004).

${ }^{12} \mathrm{M}$. A. Dahlem and S. C. Müller, "Reaction-diffusion waves in neuronal tissue and the window of cortical excitability," Ann. Phys. (Paris) 13, 442 (2004).

${ }^{13}$ J. P. Dreier, J. Woitzik, M. Fabricius, R. Bhatia, S. Major, C. Drenckhahn, T.-N. Lehmann, A. Sarrafzadeh, L. Willumsen, J. A. Hartings, O. W. Sakowitz, J. H. Seemann, A. Thieme, M. Lauritzen, and A. J. Strong, "Delayed ischemic neurological deficits after subarachnoid haemorrhage are associated with clusters of spreading depolarizations," Brain 129, 3224 (2006).

${ }^{14}$ K. A. Hossmann, "Periinfarct depolarizations," Cerebrovasc Brain Metab. Rev. 8, 195 (1996).

${ }^{15}$ Handbook of Chaos Control, 2nd completely revised and enlarged edition, edited by E. Schöll and H. G. Schuster (Wiley-VCH, Weinheim, 2008).

${ }^{16}$ K. M. Welch, "Brain hyperexcitability: The basis for antiepileptic drugs in migraine prevention," Headache 45, 25 (2005).

${ }^{17}$ O. Herreras, C. Largo, J. M. Ibarz, G. G. Somjen, and R. Martin del Rio, "Role of neuronal synchronizing mechanisms in the propagation of spreading depression in the in vivo hippocampus," J. Neurosci. 14, 7087 (1994).

${ }^{18}$ B. Larrosa, J. Pastor, L. Lopez-Aguado, and O. Herreras, "A role for glutamate and glia in the fast network oscillations preceding spreading depression," Neuroscience 141, 1057 (2006).

${ }^{19}$ H. C. Tuckwell and R. M. Miura, "A mathematical model for spreading cortical depression,” Biophys. J. 23, 257 (1978).

${ }^{20}$ H. Kager, W. J. Wadman, and G. G. Somjen, "Simulated seizures and spreading depression in a neuron model incorporating interstitial space and ion concentrations," J. Neurophysiol. 84, 495 (2000).

${ }^{21}$ B. E. Shapiro, "Osmotic forces and gap junctions in spreading depression: a computational model," J. Comput. Neurosci. 10, 99 (2001).

${ }^{22}$ J. A. Reggia and D. Montgomery, "A computational model of visual hallucinations in migraine," Comput. Biol. Med. 26, 133 (1996).

${ }^{23}$ K. Revett, E. Ruppin, S. Goodall, and J. A. Reggia, "Spreading depression in focal ischemia: A computational study," J. Cereb. Blood Flow Metab. 18, 998 (1998).

${ }^{24}$ E. Ruppin, K. Revett, E. Ofer, S. Goodall, and J. A. Reggia, "Penumbral tissue damage following acute stroke: A computational investigation," Prog. Brain Res. 121, 243 (1999).

${ }^{25}$ R. FitzHugh, "Impulses and physiological states in theoretical models of nerve membrane," Biophys. J. 1, 445 (1961).

${ }^{26}$ J. Nagumo, S. Arimoto, and S. Yoshizawa., "An active pulse transmission line simulating nerve axon.," Proc. IRE 50, 2061 (1962).

${ }^{27}$ B. Lindner, J. García-Ojalvo, A. Neiman, and L. Schimansky-Geier, "Effects of noise in excitable systems," Phys. Rep. 392, 321 (2004).

${ }^{28}$ K. Pyragas, "Continuous control of chaos by self-controlling feedback," Phys. Lett. A 170, 421 (1992).

${ }^{29}$ O. Beck, A. Amann, E. Schöll, J. E. S. Socolar, and W. Just, "Comparison of time-delayed feedback schemes for spatio-temporal control of chaos in a reaction-diffusion system with global coupling," Phys. Rev. E 66, 016213 (2002).

${ }^{30}$ N. Baba, A. Amann, E. Schöll, and W. Just, "Giant improvement of timedelayed feedback control by spatio-temporal filtering," Phys. Rev. Lett. 89, 074101 (2002).

${ }^{31}$ J. Unkelbach, A. Amann, W. Just, and E. Schöll, "Time-delay autosynchronization of the spatiotemporal dynamics in resonant tunneling diodes," Phys. Rev. E 68, 026204 (2003).

${ }^{32}$ J. Schlesner, A. Amann, N. B. Janson, W. Just, and E. Schöll, "Selfstabilization of high frequency oscillations in semiconductor superlattices by time-delay autosynchronization," Phys. Rev. E 68, 066208 (2003).

${ }^{33}$ J. Schlesner, V. Zykov, H. Engel, and E. Schöll, "Stabilization of unstable rigid rotation of spiral waves in excitable media," Phys. Rev. E 74, 046215 (2006).

${ }^{34}$ N. B. Janson, A. G. Balanov, and E. Schöll, "Delayed feedback as a means of control of noise-induced motion," Phys. Rev. Lett. 93, 010601 (2004).

${ }^{35}$ A. G. Balanov, N. B. Janson, and E. Schöll, "Control of noise-induced oscillations by delayed feedback," Physica D 199, 1 (2004).

${ }^{36}$ T. Prager, H. P. Lerch, L. Schimansky-Geier, and E. Schöll, "Increase of coherence in excitable systems by delayed feedback," J. Phys. A 40, 11045 (2007).

${ }^{37}$ B. Hauschildt, N. B. Janson, A. G. Balanov, and E. Schöll, "Noiseinduced cooperative dynamics and its control in coupled neuron models," Phys. Rev. E 74, 051906 (2006).

${ }^{38}$ P. Hövel, M. A. Dahlem, and E. Schöll, "Synchronization of noise-induced oscillations by time-delayed feedback," in Proceedings of the 19th Inter- 
national Conference on Noise and Fluctuations (ICNF-2007) (American Institute of Physics, College Park, Maryland, 2007), pp. 2074-3843.

${ }^{39}$ G. Stegemann, A. G. Balanov, and E. Schöll, "Noise-induced pattern formation in a semiconductor nanostructure," Phys. Rev. E 71, 016221 (2005).

${ }^{40}$ G. Stegemann, A. G. Balanov, and E. Schöll, "Delayed feedback control of stochastic spatiotemporal dynamics in a resonant tunneling diode," Phys. Rev. E 73, 016203 (2006).

${ }^{41}$ J. Hizanidis, A. G. Balanov, A. Amann, and E. Schöll, "Noise-induced front motion: Signature of a global bifurcation," Phys. Rev. Lett. 96, 244104 (2006).

${ }^{42}$ J. Hizanidis and E. Schöll, "Control of noise-induced spatiotemporal patterns in superlattices," Phys. Status Solidi C 5, 207 (2008).

${ }^{43}$ A. G. Balanov, V. Beato, N. B. Janson, H. Engel, and E. Schöll, "Delayed feedback control of noise-induced patterns in excitable media," Phys. Rev. E 74, 016214 (2006).

${ }^{44}$ M. Gassel, E. Glatt, and F. Kaiser, "Time-delayed feedback in a net of neural elements: Transitions from oscillatory to excitable dynamics," Fluct. Noise Lett. 7, L225 (2007).

${ }^{45} \mathrm{C}$. M. Peppiatt, C. Howarth, P. Mobbs, and D. Attwell, "Bidirectional control of CNS capillary diameter by pericytes," Nature (London) $\mathbf{4 4 3}$, 700 (2006).

${ }^{46}$ C. Iadecola, G. Yang, T. J. Ebner, and G. Chen, "Local and propagated vascular responses evoked by focal synaptic activity in cerebellar cortex," J. Neurophysiol. 78, 651 (1997).

${ }^{47}$ P. Hövel and E. Schöll, "Control of unstable steady states by time-delayed feedback methods," Phys. Rev. E 72, 046203 (2005).

${ }^{48}$ S. Schikora, P. Hövel, H. J. Wünsche, E. Schöll, and F. Henneberger, "All-optical noninvasive control of unstable steady states in a semiconductor laser," Phys. Rev. Lett. 97, 213902 (2006).

${ }^{49}$ T. Dahms, P. Hövel, and E. Schöll, "Control of unstable steady states by extended time-delayed feedback," Phys. Rev. E 76, 056201 (2007).

${ }^{50}$ A. S. Mikhailov and V. S. Zykov, "Kinematical theory of spiral waves in excitable media: comparison with numerical simulations," Physica D 52, 379 (1991).

${ }^{51}$ V. Hakim and A. Karma, "Theory of spiral wave dynamics in weakly excitable media: asymptotic reduction to a kinematic model and applications," Phys. Rev. E 60, 5073 (1999).

${ }^{52}$ E. Mihaliuk, T. Sakurai, F. Chirila, and K. Showalter, "Feedback stabilization of unstable propagating waves," Phys. Rev. E 65, 065602 (2002).

${ }^{53}$ Y. A. Kuznetsov, Elements of Applied Bifurcation Theory (Springer, New York, 1995).

${ }^{54}$ E. J. Doedel, R. C. Pfaffenroth, A. R. Chambodut, T. F. Fairgrieve, Y. A. Kuznetsov, B. E. Oldeman, B. Sandstede, and X. Wang, "AUTO 2000: Continuation and Bifurcation Software for Ordinary Differential Equations (with HomCont)" (2006).

${ }^{55} \mathrm{C}$. Nicholson and J. D. Phillips, "Ion diffusion modified by tortuosity and volume fraction in the extracellular microenvironment of the rat cerebellum," J. Physiol. (London) 321, 225 (1981)

${ }^{56}$ H. R. Wilson, Spikes, Decisions, and Actions: The Dynamical Foundations of Neuroscience (Oxford University Press, Oxford, 1999).

${ }^{57}$ M. A. Dahlem, F. M. Schneider, and E. Schöll, "Efficient control of transient wave forms to prevent spreading depolarizations," J. Theor. Biol. 251, 202 (2008).

${ }^{58}$ G. B. Ermentrout, "Neural networks as spatio-temporal pattern-forming systems," Rep. Prog. Phys. 61, 353 (1998).

${ }^{59}$ H. R. Wilson, "Simplified dynamics of human and mammalian neocortical neurons," J. Theor. Biol. 200, 375 (1999).

${ }^{60}$ J. Hizanidis, R. Aust, and E. Schöll, "Delay-induced multistability near a global bifurcation," arXiv:nlin-sys/0702002 (in press).

${ }^{61}$ Y. A. Dahlem, M. A. Dahlem, T. Mair, K. Braun, and S. C. Müller, "Extracellular potassium alters frequency and profile of retinal spreading depression waves," Exp. Brain Res. 152, 221 (2003).

${ }^{62}$ U. Heinemann and H. D. Lux, "Ceiling of stimulus induced rises in extracellular potassium concentration in the cerebral cortex of cat," Brain Res. 120, 231 (1977).

${ }^{63} \mathrm{~F}$. Schlögl, "Chemical reaction models for nonequilibrium phase transitions," Z. Phys. 253, 147 (1972).

${ }^{64}$ E. Schöll, Nonlinear Spatio-Temporal Dynamics and Chaos in Semiconductors, Nonlinear Science Series (Cambridge University Press, Cambridge, 2001), Vol. 10.

${ }^{65}$ R. H. Stokes, "The diffusion coefficients of eight uni-univalent electrolytes in aqueous solution at 25," J. Am. Chem. Soc. 72, 2243 (1950). 